\title{
LOCALLY TAUBERIAN THEOREMS FOR MEROMORPHIC FUNCTIONS OF LOWER ORDER LESS THAN ONE
}

\author{
BY \\ ALBERT EDREI( ${ }^{(1)}$
}

Introduction. Let $f(z)$ be a meromorphic function; it is assumed that the reader is familiar with the following symbols of frequent use in Nevanlinna's theory:

$$
\log ^{+}, \quad m(r, f), \quad n(r, f), \quad N(r, f), \quad T(r, f), \quad \delta(\tau, f) .
$$

The lower order $\mu$ and the order $\Lambda$ of $f(z)$ are defined by the familiar relations

$$
\liminf _{r \rightarrow \infty} \frac{\log T(r, f)}{\log r}=\mu, \quad \limsup _{r \rightarrow \infty} \frac{\log T(r, f)}{\log r}=\Lambda .
$$

In addition to these classical concepts, we shall consider the total deficiency $\Delta(f)$ of the function $f$ :

$$
\Delta(f)=\sum_{\tau} \delta(\tau, f)
$$

where the summation is to be extended to all the values $\tau$, finite or $\infty$, such that

$$
\delta(\tau, f)>0 .
$$

The number of deficient values of $f$, that is the number of distinct values of $\tau$ for which (2) holds, will be denoted by $\nu(f)(\leqq+\infty)$.

Nevanlinna's fundamental results show that

$$
\Delta(f) \leqq 2 \text {, }
$$

and it is not difficult to find functions such that equality holds in (3). However, all the theorems and examples known to the author indicate that, if $\mu$ is finite,

(i) the relation $\Delta(f)=2$ is only possible for particular values of $\mu$;

(ii) if $\Delta(f)=2$ the number of deficient values of $f(z)$ remains finite.

These remarks lead to interesting questions which may be formulated as a

DEFICIENCY PROBLEM. Let $f(z)$ be a meromorphic function of lower order $\mu<+\infty$.

I. Determine, as explicitly as possible, a function $\Xi(\mu)$ such that

$$
\Delta(f) \leqq \Xi(\mu)
$$

be sharp for all values of $\mu$.

Received by the editors February 5, 1968 and, in revised form, June 7, 1968.

( ${ }^{1}$ The research of the author was supported by a grant from the National Science Foundation GP-5019. 
II. Is it true that $\Delta(f)=\Xi(\mu)$ implies $\nu(f)<+\infty$ ? Determine as a function of $\mu$ the correct bound for $\nu(f)$.

The solution of this problem is more advanced for entire functions than for general meromorphic functions. The following is known:

Let $f(z)$ be a meromorphic function of finite lower order such that $\Delta(f)=2$, $\delta(\infty, f)=1$, and

$$
\Lambda<+\infty \text {. }
$$

Then $\mu=\Lambda=q$, where $q$ is a positive integer, and $\nu(f) \leqq q+1$.

This important result is essentially due to Pfluger [11]. [He proved it for entire functions and did not assert that $\Lambda=\mu$.] Some complements and extensions were obtained by Edrei and Fuchs [5]. One of the ideas used in the present note shows that the restriction (5) is unnecessary.

It will be noticed that, for nonintegral values of $\mu$, Pfluger's theorem tells us nothing more than $\Xi(\mu)<2$. The method developed here and in Weitsman's paper [which follows in the same issue of this Journal] yields a complete solution of the deficiency problem for entire functions of lower order $\mu<1$. More precisely, we shall prove

THEOREM A. Let $f(z)$ be a meromorphic function of lower order $\mu$ :

$$
\frac{1}{2}<\mu<1,
$$

and let the poles of $f(z)$ have maximum deficiency $(\delta(\infty, f)=1)$.

Then

$$
\Delta(f) \leqq 2-\sin \pi \mu .
$$

Moreover, if equality holds in (7), then

$$
\nu(f)=2 .
$$

Concerning the restrictions (6) we observe that:

(i) the limiting case $\mu=1$ is covered by Pfluger's theorem;

(ii) if $0 \leqq \mu \leqq(1 / 2)$, then $\nu(f)=1$. [For $\mu=0$ this is proved in [4, p. 297]. For $0<\mu \leqq 1 / 2$ the result is an obvious consequence of part $I$ of Theorem 1 of this paper.]

Theorem A is not obtained directly; it will appear as a simple corollary of several "locally tauberian" theorems which constitute the central topic of our investigation.

As far as I know, the first tauberian theorem in the theory of entire functions was discovered by Valiron in 1913 [12, p. 237].

In 1930, Hardy and Littlewood recognized the tauberian character of Valiron's statement and obtained several important generalizations. Their results are stated in terms of Laplace and Stieltjes transforms [9] and the connection with entire functions is no longer obvious. 
Recently, Edrei and Fuchs [8] returned to Valiron's original approach; they proved a tauberian theorem concerning meromorphic functions of order $\Lambda \leqq 1$ having all their zeros negative and all their poles positive.

Theorem 1 of this note is, in many respects, similar to Theorem 1 of [8]. The main difference lies in the fact that I do not impose geometrical restrictions on the position of the zeros and poles. This yields a result, which is more readily applicable, but limits me to tauberian assertions of a local nature. More precisely, my conclusions no longer hold as the relevant variable tends to $+\infty$ unrestrictedly: it must be confined to infinitely many, well-chosen intervals $\left[R_{m}^{\prime}, R_{m}^{\prime \prime}\right]$ such that

$$
\lim _{m \rightarrow \infty} R_{m}^{\prime}=+\infty, \quad \lim _{m \rightarrow \infty} \frac{R_{m}^{\prime \prime}}{R_{m}^{\prime}}=+\infty .
$$

1. Definitions and notations. Throughout this note $f(z)$ is a meromorphic function of lower order $\mu$

$$
0<\mu<1
$$

the order of $f(z)$ plays no role and may be $+\infty$. Since $\mu>0, f(z)$ cannot be rational and hence

$$
\log r=o(T(r)) \quad(r \rightarrow+\infty) .
$$

I have defined, and used elsewhere [1], [2], [3], remarkable sequences of points associated with $T(r)$. The members of these sequences are the Polya peaks, of order $\mu$, of $T(r)$ and may be introduced by the following

Definition 1. A positive sequence

$$
r_{1}, r_{2}, r_{3}, \ldots
$$

of numbers tending to $+\infty$ is said to be a sequence of Pólya peaks, of order $\mu$, of $T(r)$, if it is possible to find three positive sequences

$$
\left\{\boldsymbol{r}_{m}^{\prime}\right\}, \quad\left\{\boldsymbol{r}_{m}^{\prime \prime}\right\}, \quad\left\{\varepsilon_{m}\right\}
$$

such that, as $m \rightarrow+\infty$,

$$
r_{m}^{\prime} \rightarrow+\infty, \quad r_{m} / r_{m}^{\prime} \rightarrow+\infty, \quad r_{m}^{\prime \prime} / r_{m} \rightarrow+\infty, \quad \varepsilon_{m} \rightarrow 0,
$$

and such that the inequalities

$$
r_{m}^{\prime} \leqq t \leqq r_{m}^{\prime \prime} \quad\left(m>m_{0}\right)
$$

imply

$$
T(t) / T\left(r_{m}\right) \leqq\left(1+\varepsilon_{m}\right)\left(t / r_{m}\right)^{u} .
$$

We take for granted the fact that, if $f(z)$ is of lower order $\mu$, then $T(r)=T(r, f)$ has a sequence of Pólya peaks of order $\mu$. [A proof will be found in [2, pp. 85-86]. It might be of interest to mention that there exist sequences of Pólya peaks of every finite order $\rho$ in the interval $\mu \leqq \rho \leqq \Lambda$.] 
Let $\mathscr{E}$ denote a measurable subset of the axis $r>0$ and let $\mathscr{E}\left[r^{\prime}, r^{\prime \prime}\right]$ be the portion of $\mathscr{E}$ which lies in the interval $\left[r^{\prime}, r^{\prime \prime}\right]$.

We say that $\mathscr{E}$ has density zero if

$$
\lim _{r \rightarrow \infty} \frac{\text { meas } \mathscr{E}[0, r]}{r}=0 .
$$

We consider systematically the two quantities

$$
\limsup _{r \rightarrow \infty ; r \notin \mathscr{E}} \frac{N(r, 1 / f)}{T(r)}=u, \quad \lim _{r \rightarrow \infty ; r \notin \mathscr{E}} \frac{N(r, f)}{T(r)}=v .
$$

The set $\mathscr{E}$, which is avoided as $r \rightarrow \infty$, is always assumed to be of density zero.

If $\mathscr{E}$ is a bounded set, the formulae (1.9) reduce to

$$
1-\delta(0, f)=u, \quad 1-\delta(\infty, f)=v .
$$

For the applications which we have in mind it would be sufficient to introduce in (1.9) exceptional sets of finite measure. However, the "larger" sets of density zero create no additional complications and are in some respects more natural.

Throughout the paper we use the symbol $A$ to denote a positive absolute constant and the symbol $K$ to denote a positive constant depending on one or more parameters.

Most of our inequalities are only valid for sufficiently large values of certain parameters $m, t, r, \ldots$ We usually indicate this fact by writing, immediately after the relevant inequality, $\left(m>m_{0}\right),\left(t>t_{0}\right),\left(r>r_{0}\right), \ldots$

The quantities $A, K, m_{0}, t_{0}, r_{0}, \ldots$ are not necessarily the same ones each time they occur.

For sake of clarity, we shall sometimes indicate the parameters which are implicit in $K, m_{0}, t_{0}, r_{0}$ and write, for instance, $t_{0}(\varepsilon), K(q), \ldots$

\section{Statement and discussion of the locally tauberian results.}

THEOREM 1. Let $f(z)$ be a meromorphic function of lower order $\mu(0<\mu<1)$ and let $u$ and $v$ be defined by (1.9).

I. Then

$$
\sin ^{2} \pi \mu \leqq u^{2}+v^{2}-2 u v \cos \pi \mu .
$$

Moreover, $v \leqq \cos \pi \mu$ implies $u=1$ and $u \leqq \cos \pi \mu$ implies $v=1$.

II. Let $\left\{r_{m}\right\}$ be a sequence of Pólya peaks of order $\mu$ of $T(r)$ and let $E_{\infty}(r)$ and $E_{0}(r)$ be sets of $\theta(-\pi \leqq \theta<\pi)$ defined by

$$
E_{\infty}(r)=\left\{\theta:\left|f\left(r e^{i \theta}\right)\right|>r^{\alpha}\right\}, \quad E_{0}(r)=\left\{\theta:\left|f\left(r e^{i \theta}\right)\right|<r^{-\alpha}\right\},
$$

where $\alpha$ is an arbitrary, nonnegative constant.

Assume that equality holds in (2.1) and that

$$
u<1, \quad v<1 \text {. }
$$


Then all the following limits exist and satisfy the relations stated

$$
\begin{array}{cc}
\lim _{m \rightarrow \infty} \text { meas } E_{\infty}\left(r_{m}\right)=s(\infty)=(2 / \mu) \cos ^{-1} v & \left(0<\cos ^{-1} v \leqq \pi / 2\right), \\
\lim _{m \rightarrow \infty} \text { meas } E_{0}\left(r_{m}\right)=s(0)=(2 / \mu) \cos ^{-1} u & \left(0<\cos ^{-1} u \leqq \pi / 2\right), \\
s(0)+s(\infty)=2 \pi . &
\end{array}
$$

Moreover, there exist three positive sequences $\left\{R_{m}^{\prime}\right\},\left\{R_{m}^{\prime \prime}\right\},\left\{\tilde{\varepsilon}_{m}\right\}$ such that, as $m \rightarrow+\infty$,

$$
R_{m}^{\prime}=+\infty, \quad r_{m} / R_{m}^{\prime} \rightarrow+\infty, \quad R_{m}^{\prime \prime} / r_{m} \rightarrow+\infty, \quad \tilde{\varepsilon}_{m} \rightarrow 0,
$$

and such that

$$
R_{m}^{\prime} \leqq t \leqq R_{m}^{\prime \prime} \quad\left(m>m_{0}\right)
$$

imply

$$
\begin{gathered}
\left(t / r_{m}\right)^{\mu}\left(1+\tilde{\varepsilon}_{m}\right)^{-1} \leqq T(t) / T\left(r_{m}\right) \leqq\left(t / r_{m}\right)^{\mu}\left(1+\tilde{\varepsilon}_{m}\right), \\
u-\tilde{\varepsilon}_{m} \leqq \frac{N(t, 1 / f)}{T(t)} \leqq u+\tilde{\varepsilon}_{m}, \quad v-\tilde{\varepsilon}_{m} \leqq \frac{N(t, f)}{T(t)} \leqq v+\tilde{\varepsilon}_{m},
\end{gathered}
$$

and

$$
\mu u-\tilde{\varepsilon}_{m} \leqq \frac{n(t, 1 / f)}{T(t)} \leqq \mu u+\tilde{\varepsilon}_{m}, \quad \mu v-\tilde{\varepsilon}_{m} \leqq \frac{n(t, f)}{T(t)} \leqq \mu v+\tilde{\varepsilon}_{m} .
$$

The property of $T(t)$, expressed by (2.9), may be stated in the following equivalent form.

Let $\sigma>1$ and let

$$
I_{\sigma}\left(r_{m}\right)=\left\{t: \sigma^{-1} r_{m} \leqq t \leqq \sigma r_{m}\right\}, \quad V=V_{\sigma}\left(\left\{r_{m}\right\}\right)=\bigcup_{m=1}^{\infty} I_{\sigma}\left(r_{m}\right) .
$$

Then, for any fixed $\sigma>1$, we have

$$
\lim _{t \rightarrow \infty ; t \in V} \frac{T(\lambda t)}{T(t)}=\lambda^{\mu}
$$

uniformly in any finite interval $0<\lambda_{1} \leqq \lambda \leqq \lambda_{2}$.

The relations (2.10) and (2.11) may be restated in a similar manner. For instance, the last of the relations (2.11) is equivalent to the following assertion: for any fixed $\sigma>1$,

$$
\lim _{t \rightarrow \infty: t \in V} \frac{n(t, f)}{T(t)}=\mu v \quad\left(V=V_{\sigma}\left(\left\{r_{m}\right\}\right)\right)
$$

The statements concerning (2.13) and (2.14) may be considered as "locally tauberian" because they only assert the existence of the limits in the vicinity $V_{\sigma}\left(\left\{r_{m}\right\}\right)$ of the Pólya peaks $\left\{r_{m}\right\}$. They are no longer true if we omit the restriction 
$t \in V$ : there exist functions $\left({ }^{2}\right)$ satisfying all the conditions of part II of Theorem 1 and such that each of the ratios

$$
\frac{T(\lambda t)}{T(t)}, \frac{N(t, 1 / f)}{T(t)}, \frac{N(t, f)}{T(t)}, \frac{n(t, 1 / f)}{T(t)}, \frac{n(t, f)}{T(t)},
$$

has no limit as $t \rightarrow+\infty[\lambda \neq 0, \lambda$ fixed $]$.

Theorem 1 is unsatisfactory in one respect: it provides no information concerning the angular distribution of the zeros and poles whose moduli are, in an obvious sense, determined by (2.11). A. Weitsman has filled this gap and developed an ingenious method which yields (in asymptotic form) the arguments of almost all these zeros and poles. The asymptotic evaluation of $f(z)$ which may be derived from this additional knowledge is essential in the proof of Theorem A.

The deficiency problem stated in the Introduction appears to be simpler for entire functions than for general meromorphic functions. The simplification depends on the simultaneous consideration of functions and derivatives.

Let $g(z)$ be an entire function of finite order, or more generally a meromorphic function of finite order such that $\delta(\infty, g)=1$, it is well known [13, p. 22] that the total deficiency $\Delta(g)$ satisfies the condition $\Delta(g) \leqq 1+\delta\left(0, g^{\prime}\right)$.

If the lower order of $g$ is finite, its order may be $+\infty$ and the preceding inequality must be weakened by the introduction of exceptional sets, of finite measure.

My Theorem 1 is not affected by the presence of such exceptional sets so that it is possible to investigate its implications concerning $g^{\prime}(z)$, when $g(z)$ is of lower order $\mu<1$.

Put $g^{\prime}(z)=f(z)$ and

$$
\begin{gathered}
T\left(t, g^{\prime}\right)=T(t, f)=T_{1}(t), \quad T(t, g)=T(t), \\
u\left(g^{\prime}\right)=\lim _{r \rightarrow \infty ; r \notin \mathscr{E}} \sup _{\frac{N}{1}\left(r, 1 / g^{\prime}\right)}, \quad v\left(g^{\prime}\right)=\lim _{r \rightarrow \infty ; r \notin \mathscr{E}} \frac{N\left(r, g^{\prime}\right)}{T_{1}(r)},
\end{gathered}
$$

where the set $\mathscr{E}$, which depends on $T(t)$, is of finite measure (and hence of density zero).

Using these notations, I deduce from Theorem 1:

THEOREM 2. Let $g(z)$ be a meromorphic function of lower order $\mu, \frac{1}{2}<\mu<1$, and let $\delta(\infty, g)=1$.

I. Then

$$
\Delta(g) \leqq 2-\sin \pi \mu .
$$

II. Assume that equality holds in (2.17).

Then

$$
u\left(g^{\prime}\right)=\sin \pi \mu, \quad v\left(g^{\prime}\right)=0,
$$

$\left({ }^{2}\right)$ Such functions have been constructed by $\mathrm{H}$. Silverman in his doctoral dissertation [Syracuse University]. His examples have a prescribed lower order $\mu(0<\mu<1)$ and a prescribed order $\Lambda$, chosen arbitrarily in the range $\mu<\Lambda \leqq 1$. It is not certain that it is also possible to find similar functions such that $\mu<1<\Lambda$. 
and, if $\left\{r_{m}\right\}$ is a sequence of Polya peaks (of order $\mu$ ) of $T_{1}(r)$, there exist three positive sequences $\left\{R_{m}^{\prime}\right\},\left\{R_{m}^{\prime \prime}\right\},\left\{\tilde{\varepsilon}_{m}\right\}$ satisfying the conditions (2.7) and such that

$$
R_{m}^{\prime} \leqq t \leqq R_{m}^{\prime \prime} \quad\left(m>m_{0}\right)
$$

implies

$$
\begin{gathered}
\left(t / r_{m}\right)^{\mu}\left(1+\tilde{\varepsilon}_{m}\right)^{-1} \leqq T_{1}(t) / T_{1}\left(r_{m}\right) \leqq\left(1+\tilde{\varepsilon}_{m}\right)\left(t / r_{m}\right)^{\mu}, \\
-\tilde{\varepsilon}_{m}+\sin \pi \mu \leqq N\left(t, 1 / g^{\prime}\right) / T_{1}(t) \leqq \tilde{\varepsilon}_{m}+\sin \pi \mu, \quad N\left(t, g^{\prime}\right) / T_{1}(t) \leqq \tilde{\varepsilon}_{m}, \\
-\tilde{\varepsilon}_{m}+\mu \sin \pi \mu \leqq n\left(t, 1 / g^{\prime}\right) / T_{1}(t) \leqq \tilde{\varepsilon}_{m}+\mu \sin \pi \mu, \quad n\left(t, g^{\prime}\right) / T_{1}(t) \leqq \tilde{\varepsilon}_{m},
\end{gathered}
$$

and

$$
\left(1+\tilde{\varepsilon}_{m}\right)^{-1} \leqq T(t) / T_{1}(t) \leqq\left(1+\tilde{\varepsilon}_{m}\right) .
$$

Since (2.20) and (2.23) imply

$$
\left(1+\tilde{\varepsilon}_{m}\right)^{-3}\left(t / r_{m}\right)^{\mu} \leqq T(t) / T\left(r_{m}\right) \leqq\left(1+\tilde{\varepsilon}_{m}\right)^{3}\left(t / r_{m}\right)^{\mu},
$$

we have

COROLlary 2.1. A sequence of Pólya peaks (of order $\mu$ ) of $T_{1}(r)$ is also a sequence of Pólya peaks (of order $\mu$ ) of $T(r)$.

One last remark concerns the possibility of replacing, in the statements of Theorems A, 1 and 2, the lower order $\mu$ by the order $\Lambda$.

The resulting theorems are true because an inspection of our proofs shows that the lower order $\mu$ is only used to assert the existence of a sequence of Pólya peaks of order $\mu$. Hence it is possible to restate our theorems with $\mu$ replaced by any $\rho$ $(<1)$ such that there exists a sequence of Pólya peaks of order $\rho$, of $T(r)$. In view of the remark in $\S 1$ (following formula (1.7)), any $\rho$ satisfying the conditions

$$
\mu \leqq \rho \leqq \Lambda, \quad \rho<1,
$$

is acceptable.

The following Corollary 1.1 is an easy consequence of these observations.

Corollary 1.1. Let $f(z)$ be a meromorphic function of order $\Lambda<1$ and lower order $\mu$. Assume that the quantities $u$ and $v$ defined by (1.9) satisfy the conditions

$$
\sin ^{2} \mu \Lambda=u^{2}+v^{2}-2 u v \cos \pi \Lambda, \quad u<1, v<1 .
$$

Then $\mu=\Lambda$.

Minor modifications of an argument of Edrei and Fuchs [8, pp. 351-352] readily yield this corollary. For sake of completeness a brief sketch of its proof is given in $\$ 11$.

3. Influence of the exceptional set. Since $\mathscr{E}$ is of density zero, there exists a positive sequence $\left\{\eta_{m}\right\}$ such that

$$
\sup _{r_{m} \leqq t} \frac{\text { meas } \mathscr{E}[0, t]}{t}<\eta_{m}, \quad \lim _{m \rightarrow \infty} \eta_{m}=0 .
$$


Consider the sequences which appear in (1.5) and let

$$
\rho_{m}=\min \left\{r_{m}^{\prime \prime}, r_{m} /\left(\eta_{m}\right)^{1 / 2}\right\},
$$

so that, as $m \rightarrow \infty$, we have

$$
\rho_{m} / r_{m} \rightarrow+\infty
$$

and

$$
\text { meas } \mathscr{E}\left[r_{m}^{\prime}, \rho_{m}\right]<\eta_{m} \rho_{m} \leqq\left(\eta_{m}\right)^{1 / 2} r_{m} .
$$

Assume further that $\left\{\gamma_{m}\right\}$ is a real sequence such that

$$
\eta<\gamma_{m}<\pi-\eta \quad(0<2 \eta<\pi),
$$

with $\eta$ fixed.

We now set

$$
P(t, r, \gamma)=\frac{1}{\pi} \frac{r \sin \gamma}{t^{2}+r^{2}+2 t r \cos \gamma},
$$

(this notation will be used throughout the paper) and prove that

$$
J_{m}=\int_{\mathscr{E}\left[r_{m}^{\prime}, \rho_{m}\right]} T(t) P\left(t, r_{m}, \gamma_{m}\right) d t \leqq \frac{\left(\eta_{m}\right)^{1 / 2}}{\sin \eta} T\left(r_{m}\right) \quad\left(m>m_{0}\right) .
$$

Notice that

$$
\begin{aligned}
t^{2}+r^{2}+2 t r \cos \gamma & =(t+r \cos \gamma)^{2}+r^{2} \sin ^{2} \gamma \\
& =(r+t \cos \gamma)^{2}+t^{2} \sin ^{2} \gamma \geqq(1 / 2)\left(t^{2}+r^{2}\right) \sin ^{2} \gamma,
\end{aligned}
$$

and hence

$$
J_{m} \leqq \frac{2 r_{m}}{\pi \sin \gamma_{m}} \int_{\mathscr{8}\left[r_{m}^{\prime}, \rho_{m}\right]} \frac{T(t)}{t^{2}+r_{m}^{2}} d t
$$

In view of (1.7)

$$
J_{m} \leqq \frac{T\left(r_{m}\right)}{\sin \gamma_{m}} \int_{\mathscr{E}\left[r_{m}^{\prime}, \rho_{m}\right]} \frac{\left(t / r_{m}\right)^{\mu}}{\left(t / r_{m}\right)^{2}+1} d\left(\frac{t}{r_{m}}\right) \quad\left(m>m_{0}\right) .
$$

The change of variable $x=\left(t / r_{m}\right)$ transforms the set $\mathscr{E}\left[r_{m}^{\prime}, \rho_{m}\right]$ into a set $\mathscr{E}_{m}$ which, in view of (3.4), has a measure not exceeding $\left(\eta_{m}\right)^{1 / 2}$. Hence (3.7) is an obvious consequence of (3.8) and (3.5).

\section{The main lemmas.}

LEMMA 1. Let $f(z)$ be a meromorphic function of lower order $\mu(0<\mu<1)$ and let $E_{\infty}(r)$ and $\left\{r_{m}\right\}$ be defined as in Theorem 1 .

I.et

$$
\gamma(r)=\frac{1}{2} \text { meas } E_{\infty}(r), \quad \gamma\left(r_{m}\right)=\gamma_{m}
$$

and assume that, for a fixed $\eta$,

$$
\eta<\gamma_{m}<\pi-\eta \quad\left(m>m_{0}, 0<2 \eta<\pi\right) .
$$


Then there exist two sequences $\left\{\rho_{m}^{\prime}\right\},\left\{\rho_{m}^{\prime \prime}\right\}$ such that, as $m \rightarrow \infty$,

$$
\rho_{m}^{\prime} \rightarrow+\infty, \frac{r_{m}}{\rho_{m}^{\prime}} \rightarrow+\infty, \quad \frac{\rho_{m}^{\prime \prime}}{r_{m}} \rightarrow+\infty \quad\left(\rho_{m}^{\prime}=r_{m}^{\prime}, 2 \rho_{m}^{\prime \prime} \leqq r_{m}^{\prime \prime}\right),
$$

and such that if

$$
\mathscr{L}_{m}=\left[\rho_{m}^{\prime}, \rho_{m}^{\prime \prime}\right]-\mathscr{E}\left[\rho_{m}^{\prime}, \rho_{m}^{\prime \prime}\right]
$$

we have (with the notation (3.6))

$$
\begin{aligned}
T\left(r_{m}\right) \leqq & \int_{\mathscr{L}_{m}} N(t, 1 / f) P\left(t, r_{m}, \gamma_{m}\right) d t \\
& +\int_{\mathscr{L}_{m}} N(t, f) P\left(t, r_{m}, \pi-\gamma_{m}\right) d t+o\left(T\left(r_{m}\right)\right) \quad(m \rightarrow+\infty)
\end{aligned}
$$

Proof. Put

$$
\begin{aligned}
& N_{0}(t)=\int_{0}^{t} \frac{n(x, 1 / f)-n(0,1 / f)}{x} d x, \\
& N_{\infty}(t)=\int_{0}^{t} \frac{n(x, f)-n(0, f)}{x} d x .
\end{aligned}
$$

Then, as I have shown elsewhere [2, pp. 89-91], the inequalities $R \geqq 2 r$ and

$$
0<\gamma(r)<\pi,
$$

imply, for all such $\gamma(r)$,

$$
\begin{aligned}
T(r) \leqq & \int_{0}^{R} N_{0}(t) P(t, r, \gamma(r)) \cdot d t+\int_{0}^{R} N_{\infty}(t) P(t, r, \pi-\gamma(r)) d t \\
& +A \frac{r}{R} T(2 R)+K \log r \quad(r \rightarrow \infty),
\end{aligned}
$$

where $A$ is an absolute constant and $K$ depends only on $f$ and $\alpha$. We set in this inequality

$$
r=r_{m}, \quad R=\rho_{m} / 2=\rho_{m}^{\prime \prime} .
$$

The quantity $\rho_{m}$, which depends on the exceptional set $\mathscr{E}$, is defined by (3.2); hence $2 \rho_{m}^{\prime \prime} \leqq r_{m}^{\prime \prime}$. In view of (4.2), (4.4) and (1.2), we deduce from (4.8)

$$
\begin{aligned}
& T\left(r_{m}\right) \leqq N_{0}\left(\rho_{m}^{\prime}\right) \int_{0}^{\rho_{m}^{\prime}} P\left(t, r_{m}, \gamma_{m}\right) d t+N_{\infty}\left(\rho_{m}^{\prime}\right) \int_{0}^{\rho_{m}^{\prime}} P\left(t, r_{m}, \pi-\gamma_{m}\right) d t \\
&+2 A \frac{r_{m}}{\rho_{m}} T\left(\rho_{m}\right)+o\left(T\left(r_{m}\right)\right)+\int_{\mathscr{L}_{m}} N_{0}(t) P\left(t, r_{m}, \gamma_{m}\right) d t \\
&+\int_{\mathscr{L}_{m}} N_{\infty}(t) P\left(t, r_{m}, \pi-\gamma_{m}\right) d t \\
&+2 \int_{\mathscr{\delta}_{\left[\rho_{m}^{\prime}, \rho_{m}^{m}\right]}} T(t)\left[P\left(t, r_{m}, \gamma_{m}\right)+P\left(t, r_{m}, \pi-\gamma_{m}\right)\right] d t \\
& \quad\left(m \rightarrow \infty, \rho_{m}^{\prime}=r_{m}^{\prime}\right) .
\end{aligned}
$$


By (3.7), the integral over $\mathscr{E}\left[\rho_{m}^{\prime}, \rho_{m}^{\prime \prime}\right]$ is $o\left(T\left(r_{m}\right)\right)$ and therefore may be absorbed by the term $o\left(T\left(r_{m}\right)\right)$ which is already present.

Similarly, by (1.1), (1.7), (3.2) and (3.3)

$$
\frac{r_{m}}{\rho_{m}} T\left(\rho_{m}\right) \leqq 2 \frac{r_{m}}{\rho_{m}}\left(\frac{\rho_{m}}{r_{m}}\right)^{\mu} T\left(r_{m}\right)=o\left(T\left(r_{m}\right)\right) \quad(m \rightarrow+\infty) .
$$

The terms, in (4.9), involving $N_{0}\left(\rho_{m}^{\prime}\right)$ and $N_{\infty}\left(\rho_{m}^{\prime}\right)$ may be estimated immediately since each one of them is dominated by

$$
2 T\left(\rho_{m}^{\prime}\right) \int_{0}^{\rho_{m}^{\prime}} \frac{r_{m}}{t^{2}+r_{m}^{2}-2 t r_{m}} d t \leqq A T\left(\rho_{m}^{\prime}\right) \frac{\rho_{m}^{\prime}}{r_{m}}=o\left(T\left(r_{m}\right)\right) \quad(m \rightarrow \infty) .
$$

Finally, we observe that, for $t \geqq 1$

$$
N_{0}(t) \leqq N(t, 1 / f), \quad N_{\infty}(t) \leqq N(t, f),
$$

and since all the points of $\mathscr{L}_{m}$ exceed one (for $m>m_{0}$ ), we may in (4.9) replace $N_{0}(t), N_{\infty}(t)$ by $N(t, 1 / f), N(t, f)$, respectively.

The inequality (4.5) is now obvious if we take into account (4.10) and (4.11).

Lemma 1, which we have thus proved, will be first used to obtain the following lemma, fundamental in this investigation. I proved a similar lemma in [2, pp. 8794]; the new form is slightly more general because of the presence of exceptional sets.

LEMMA 2. Let $f(z),\left\{r_{m}\right\},\left\{\gamma_{m}\right\}$ have the same meaning as in Lemma 1 and let $u$ and $v$ be defined by (1.9). [The restrictions (4.2) are omitted.]

Then, if $\beta$ is any point of accumulation of $\left\{\gamma_{m}\right\}$, we have

$$
\sin \pi \mu \leqq u \sin \beta \mu+v \sin (\pi-\beta) \mu .
$$

Proof. Assume first that $\beta \neq 0, \beta \neq \pi$ and confine $m$ to a sequence of integers such that

$$
\gamma_{m} \rightarrow \beta \quad(m \rightarrow+\infty) .
$$

On the sets $\mathscr{L}_{m}$ we have, by definition,

$$
N(t, 1 / f) \leqq\left(u+\xi_{m}\right) T(t),
$$

where $\left\{\xi_{m}\right\}$ is a suitable sequence tending to zero.

Hence, by (1.7),

$$
\begin{aligned}
\frac{1}{T\left(r_{m}\right)} \int_{\mathscr{L}_{m}} N\left(t, \frac{1}{f}\right) P\left(t, r_{m}, \gamma_{m}\right) d t \\
\quad \leqq\left(u+\zeta_{m}\right) \int_{\rho_{m}^{\prime}}^{\rho_{m}^{*}}\left(1+\varepsilon_{m}\right)\left(\frac{t}{r_{m}}\right)^{\mu} P\left(t, r_{m}, \gamma_{m}\right) d t \\
\quad \leqq\left(u+\zeta_{m}\right)\left(1+\varepsilon_{m}\right) \int_{0}^{+\infty} x^{\mu} P\left(x, 1, \gamma_{m}\right) d x=\left(u+\xi_{m}\right)\left(1+\varepsilon_{m}\right) \frac{\sin \gamma_{m} \mu}{\sin \pi \mu},
\end{aligned}
$$


where the explicit evaluation of the last integral is a simple consequence of contour integration.

This inequality and a similar one involving $N(t, f), v$ and $\pi-\gamma_{m}$ (instead of $N(t, 1 / f), u$ and $\left.\gamma_{m}\right)$, used in (4.5), yield (4.12) provided $\beta \neq 0$ and $\beta \neq \pi$. The latter restrictions are easily removed. Assume, for instance that, as $m \rightarrow+\infty$ by a suitable set of integers,

$$
\gamma_{m} \rightarrow \pi=\beta .
$$

This means that $1 /\left|f\left(r_{m} e^{i \theta}\right)\right|>1$ for a set of values of $\theta$ of measure $\zeta_{m} \rightarrow 0$.

By a lemma proved elsewhere [7, Lemma III, p. 322], we have

$$
m\left(r_{m}, \frac{1}{f}\right) \leqq \frac{11 R_{m}^{*}}{R_{m}^{*}-r_{m}} T\left(R_{m}^{*}, \frac{1}{f}\right) \zeta_{m}\left(1+\log ^{+} \frac{1}{\zeta_{m}}\right) \quad\left(m>m_{0}\right)
$$

where we may choose $R_{m}^{*}=2 r_{m}$. In view of (1.7), this leads to

$$
m\left(r_{m}, \frac{1}{f}\right)=o\left(T\left(r_{m}\right)\right) \quad(m \rightarrow+\infty) .
$$

Let $\varepsilon(>0)$ be given. Since $\mathscr{E}$ is of density zero, each interval $\left[r_{m}, r_{m}(1+\varepsilon)\right]$ contains a point $r_{m}^{*} \notin \mathscr{E}$, provided $m$ is large enough. Hence, by the first fundamental theorem of Nevanlinna

$$
\begin{aligned}
T\left(r_{m}\right) & \leqq N\left(r_{m}, \frac{1}{f}\right)+o\left(T\left(r_{m}\right)\right) \\
& \leqq N\left(r_{m}^{*}, \frac{1}{f}\right)+o\left(T\left(r_{m}\right)\right) \leqq(u+\varepsilon) \frac{T\left(r_{m}^{*}\right)}{T\left(r_{m}\right)} T\left(r_{m}\right)+o\left(T\left(r_{m}\right)\right) .
\end{aligned}
$$

After dividing by $T\left(r_{m}\right)$ we use (1.7) and let $m \rightarrow+\infty$ by suitable values. This yields

$$
1 \leqq(u+\varepsilon)(1+\varepsilon)^{\mu},
$$

and since $\varepsilon(>0)$ is arbitrary, we obtain $u \geqq 1$, which is precisely (4.12) for $\beta=\pi$. The case $\beta=0$ is analogous and requires the consideration of $m(r, f)$ instead of $m(r, 1 / f)$. The proof of Lemma 2 is complete.

5. Proof of assertion I of Theorem 1. The inequality (2.1) is an immediate consequence of (4.12) and of the Cauchy-Schwarz inequality:

$$
\begin{aligned}
\sin \pi \mu & \leqq(u-v \cos \pi \mu) \sin \beta \mu+v \sin \pi \mu \cos \beta \mu \\
& \leqq\left((u-v \cos \pi \mu)^{2}+v^{2} \sin ^{2} \pi \mu\right)^{1 / 2}=\left(u^{2}+v^{2}-2 u v \cos \pi \mu\right)^{1 / 2} .
\end{aligned}
$$

We have thus proved (2.1) and examine now the implications of

$$
v \leqq \cos \pi \mu .
$$

[Since $v \geqq 0$, this is only possible if $\mu \leqq \frac{1}{2}$.] 
As shown by Edrei and Fuchs [6, pp. 241-242] (4.12) and (5.2) yield

$$
\begin{aligned}
u \sin \beta \mu & \geqq \sin \pi \mu-\cos \pi \mu \sin (\pi-\beta) \mu \\
& \geqq \sin \pi \mu \cos (\pi-\beta) \mu-\cos \pi \mu \sin (\pi-\beta) \mu=\sin \beta \mu,
\end{aligned}
$$

where $\beta \neq 0$ (by (1.1), (4.12) and (5.2)). Hence (5.2) implies $u \geqq 1$. We complete the proof of assertion I Theorem 1 by noticing that the roles of $u$ and $v$ may be exchanged.

6. Behavior of $T(r)$ if equality holds in (2.1). If equality holds in (2.1) it must also hold in (5.1) and hence strict inequality is precluded in our application of the Cauchy-Schwarz inequality. As is well known, this implies the existence of some factor $\zeta$ such that

$$
u-v \cos \pi \mu=\zeta \sin \beta \mu, \quad v \sin \pi \mu=\zeta \cos \beta \mu .
$$

Returning to (5.1), in which equality must hold everywhere, we find $\zeta=\sin \pi \mu$, and hence

$$
v=\cos \beta \mu .
$$

Since $\beta$ is any one of the limit-points of $\left\{\gamma_{m}\right\}$, we must have $0 \leqq \beta \leqq \pi$ and consequently

$$
0 \leqq \beta \mu \leqq \pi .
$$

Now (2.3) and the definition of $v$ yield $0 \leqq v<1$ and hence (6.1) shows that (6.2) may be replaced by the sharper inequalities

$$
0<\beta \mu \leqq \pi / 2 \text {. }
$$

From (6.1) and (6.3) we deduce

$$
\beta=(1 / \mu) \cos ^{-1} v \quad\left(0<\cos ^{-1} v \leqq \pi / 2\right),
$$

which determines uniquely $\beta$. This shows that the sequence $\left\{\gamma_{m}\right\}$ has a single point of accumulation; it is therefore convergent and (2.4) follows.

If we rewrite (5.1) as

$$
\sin \pi \mu \leqq(v-u \cos \pi \mu) \sin (\pi-\beta) \mu+u \sin \pi \mu \cos (\pi-\beta) \mu,
$$

the arguments which lead to (6.4) now yield

$$
\pi-\beta=(1 / \mu) \cos ^{-1} u \quad\left(0<\cos ^{-1} u \leqq \pi / 2\right) .
$$

Let $h(z)=1 / f(z)$ and notice that the Pólya peaks of $T(r, h)$ coincide with those of $T(r, f)$ since

$$
T(r, f) \sim T(r, h) \quad(r \rightarrow+\infty) .
$$

For $h(z)$, the formula corresponding to $(2.4)$ is

$$
\lim _{m \rightarrow \infty} \text { meas } E_{0}\left(r_{m}\right)=(2 / \mu) \cos ^{-1} u
$$


where

$$
E_{0}(r)=\left\{\theta:\left|h\left(r e^{i \theta}\right)\right| \geqq r^{\alpha}\right\} .
$$

We have thus proved (2.5) and, in view of (6.5), we also obtain (2.6).

In the remainder of this section we prove

LEMMA 3. The conditions

$$
u^{2}+v^{2}-2 u v \cos \pi \mu=\sin ^{2} \pi \mu, \quad u<1, v<1,
$$

imply the following property of $T(r)$ :

For every fixed $\lambda(0<\lambda<+\infty)$

$$
\lim _{m \rightarrow \infty} \frac{T\left(\lambda r_{m}\right)}{T\left(r_{m}\right)}=\lambda^{\mu}
$$

Proof. Notice first that (1.7) implies

$$
\limsup _{m \rightarrow \infty} \frac{T\left(\lambda r_{m}\right)}{T\left(r_{m}\right)} \leqq \lambda^{\mu}
$$

Hence, if we assume (6.7) to be false, there exist $\lambda(0<\lambda<+\infty)$ and $\omega(0<\omega<1)$ such that

$$
T\left(\lambda r_{m}\right) / T\left(r_{m}\right)<\omega^{2 \mu} \lambda^{\mu} \quad\left(m>m_{0}\right),
$$

as $m \rightarrow+\infty$ by a suitable sequence of integers. If necessary, we renumber the points $r_{m}$ and assume that (6.9) holds for all $m>m_{0}$.

We now consider Lemma 1 , which is certainly applicable because, by (6.4) and (6.5),

$$
0<\beta<\pi
$$

and hence it is possible to find a suitable $\eta$ such that the condition (4.2) be satisfied. Given $\varepsilon(0<\varepsilon<1)$, we deduce from (4.5) and (1.9)

$$
\begin{aligned}
(1-\varepsilon) T\left(r_{m}\right) \leqq & (u+\varepsilon) \int_{\rho_{m}^{\prime}}^{\rho_{m}^{*}} T(t) P\left(t, r_{m}, \gamma_{m}\right) d t \\
& +(v+\varepsilon) \int_{\rho_{m}^{\prime}}^{\rho_{m}^{*}} T(t) P\left(t, r_{m}, \pi-\gamma_{m}\right) d t \quad\left(m>m_{0}(\varepsilon)\right) .
\end{aligned}
$$

The range of integration $\left[\rho_{m}^{\prime}, \rho_{m}^{\prime \prime}\right]$ will be divided in three subintervals

$$
\left[\rho_{m}^{\prime}, \omega \lambda r_{m}\right], \quad\left[\omega \lambda r_{m}, \lambda r_{m}\right], \quad\left[\lambda r_{m}, \rho_{m}^{\prime \prime}\right]
$$

denoted, respectively, by $I_{1}\left(r_{m}\right), I_{2}\left(r_{m}\right), I_{3}\left(r_{m}\right)$.

Our aim is to verify that, in (6.11), the portion of the integrals extended over $I_{2}\left(r_{m}\right)$ is too small to allow equality to hold in (4.12). 
Since $T(t)$ is not decreasing, (6.9) yields

$$
\begin{aligned}
\frac{1}{T\left(r_{m}\right)} \int_{I_{2}\left(r_{m}\right)} T(t) P\left(t, r_{m}, \gamma_{m}\right) d t & \leqq \omega^{2 \mu} \lambda^{\mu} \int_{I_{2}\left(r_{m}\right)} P\left(t, r_{m}, \gamma_{m}\right) d t \\
& =\omega^{2 \mu} \lambda^{\mu} \int_{\omega \lambda}^{\lambda} P\left(x, 1, \gamma_{m}\right) d x,
\end{aligned}
$$

and a similar relation holds with $\gamma_{m}$ replaced by $\pi-\gamma_{m}$.

A direct application of (1.7) and the observation that

$$
\rho_{m}^{\prime}=r_{m}^{\prime}, \quad 2 \rho_{m}^{\prime \prime}=\rho_{m} \leqq r_{m}^{\prime \prime},
$$

yield

$$
\begin{aligned}
\frac{1}{T\left(r_{m}\right)}\left\{\int_{I_{1}\left(r_{m}\right)}+\int_{I_{3}\left(r_{m}\right)}\right\} T(t) P\left(t, r_{m}, \gamma_{m}\right) d t \\
\leqq\left(1+\varepsilon_{m}\right)\left\{\int_{0}^{\omega \lambda}+\int_{\lambda}^{+\infty}\right\} x^{\mu} P\left(x, 1, \gamma_{m}\right) d x
\end{aligned}
$$

Combining (6.12) and (6.13), we find

$$
\begin{aligned}
\frac{1}{T\left(r_{m}\right)} \int_{\rho_{m}^{\prime}}^{\rho_{m}^{*}} T(t) P\left(t, r_{m}, \gamma_{m}\right) d t \leqq & \left(1+\varepsilon_{m}\right) \int_{0}^{+\infty} x^{\mu} P\left(x, 1, \gamma_{m}\right) d x \\
& -\int_{\omega \lambda}^{\lambda}\left(x^{\mu}-\omega^{2 \mu} \lambda^{\mu}\right) P\left(x, 1, \gamma_{m}\right) d x,
\end{aligned}
$$

and a similar relation holds with $\gamma_{m}$ replaced by $\pi-\gamma_{m}$. Hence (6.11) implies

$$
\begin{aligned}
(1-\varepsilon) \leqq & (u+\varepsilon)\left(1+\varepsilon_{m}\right) \frac{\sin \left(\gamma_{m} \mu\right)}{\sin \pi \mu}+(v+\varepsilon)\left(1+\varepsilon_{m}\right) \frac{\sin \left(\pi-\gamma_{m}\right) \mu}{\sin \pi \mu} \\
& -(u+\varepsilon) \int_{\omega \lambda}^{\lambda}\left(x^{\mu}-\omega^{2 \mu} \lambda^{\mu}\right) P\left(x, 1, \gamma_{m}\right) d x \\
& -(v+\varepsilon) \int_{\omega \lambda}^{\lambda}\left(x^{\mu}-\omega^{2 \mu} \lambda^{\mu}\right) P\left(x, 1, \pi-\gamma_{m}\right) d x \quad\left(m>m_{0}\right) .
\end{aligned}
$$

In the interval $[\omega \lambda, \lambda]$, we have, since $0<\omega<1$,

$$
0<\omega^{\mu} \lambda^{\mu}\left(1-\omega^{\mu}\right) \leqq x^{\mu}-\omega^{2 \mu} \lambda^{\mu},
$$

and hence as $m \rightarrow+\infty$, (6.15) yields

$$
(1-\varepsilon) \leqq(u+\varepsilon) \frac{\sin \beta \mu}{\sin \pi \mu}+(v+\varepsilon) \frac{\sin (\pi-\beta) \mu}{\sin \pi \mu}
$$

$$
\begin{aligned}
& -\omega^{\mu} \lambda^{\mu}\left(1-\omega^{\mu}\right)\left\{(u+\varepsilon) \int_{\omega \lambda}^{\lambda} P(x, 1, \beta) d x\right. \\
& \left.+(v+\varepsilon) \int_{\omega \lambda}^{\lambda} P(x, 1, \pi-\beta) d x\right\} \quad(0<\beta<\pi) .
\end{aligned}
$$


We now let $\varepsilon \rightarrow 0$, in (6.16), and notice that the inequality

$$
u+v>0
$$

(which follows from (4.12) and (1.1)) leads to

$$
\sin \pi \mu<u \sin \beta \mu+v \sin (\pi-\beta) \mu .
$$

In view of (5.1), the relations (6.18) and (6.6) are incompatible. The origin of this contradiction is to be found in (6.9). Hence (6.7) must hold and Lemma 3 is proved.

7. Proof of (2.9). As might be expected, the relation (6.7) and the fact that $T(t)$ is nondecreasing enable us to obtain uniform upper and lower bounds for $T(t) / T\left(r_{m}\right)$, provided $t / r_{m}$ is suitably restricted. For sake of completeness, I sketch, very briefly, a proof of the following

LEMMA 4. Let $\phi(t)$ be a nondecreasing function such that

$$
\lim _{m \rightarrow \infty} \frac{\phi\left(\lambda r_{m}\right)}{\phi\left(r_{m}\right)}=\lambda^{\mu}
$$

for every fixed $\lambda(0<\lambda<+\infty)$.

Then, given $B>1$ and $\varepsilon(0<\varepsilon<1)$, it is possible to determine $m_{0}=m_{0}(B, \varepsilon)$ such that the inequalities

$$
B^{-1} r_{m} \leqq t \leqq B r_{m}, \quad\left(m>m_{0}\right)
$$

imply

$$
(1+\varepsilon)^{-1}\left(t / r_{m}\right)^{\mu} \leqq \phi(t) / \phi\left(r_{m}\right) \leqq(1+\varepsilon)\left(t / r_{m}\right)^{\mu} .
$$

It is also possible to find three sequences $\left\{R_{m}^{\prime}\right\},\left\{R_{m}^{\prime \prime}\right\},\left\{\tilde{\varepsilon}_{m}\right\}$, satisfying the conditions

$$
R_{m}^{\prime} \rightarrow+\infty, \quad r_{m} / R_{m}^{\prime} \rightarrow+\infty, \quad R_{m}^{\prime \prime} / r_{m} \rightarrow+\infty, \quad \tilde{\varepsilon}_{m} \rightarrow 0,
$$

and such that

$$
R_{m}^{\prime} \leqq t \leqq R_{m}^{\prime \prime} \quad\left(m>m_{0}\right)
$$

implies

$$
\left(1+\tilde{\varepsilon}_{m}\right)^{-1}\left(t / r_{m}\right)^{\mu} \leqq \phi(t) / \phi\left(r_{m}\right) \leqq\left(1+\tilde{\varepsilon}_{m}\right)\left(t / r_{m}\right)^{\mu} .
$$

Proof. Set $\lambda=B^{1 / k}$ and let the positive integer $k$ be so large that

$$
\lambda^{\mu} \leqq(1+\varepsilon)^{1 / 2} .
$$

We now determine $m_{0}$ so that $m>m_{0}$ implies

$$
\lambda^{j u}(1+\varepsilon)^{-1 / 2} \leqq \phi\left(\lambda^{j} r_{m}\right) / \phi\left(r_{m}\right) \leqq \lambda^{j u}(1+\varepsilon)^{1 / 2}
$$

for all integers $j$ such that

$$
-k \leqq j \leqq k
$$


If $t$ is in an interval determined by (7.2), we have

$$
r_{m} \lambda^{j} \leqq t \leqq r_{m} \lambda^{j+1},
$$

for some suitable integer $j(-k \leqq j \leqq k-1)$.

Since $\phi(t)$ is nondecreasing, we obtain, in view of (7.8), (7.10) and (7.7)

$$
\frac{\phi(t)}{\phi\left(r_{m}\right)} \geqq \frac{\phi\left(\lambda^{j} r_{m}\right)}{\phi\left(r_{m}\right)} \geqq \lambda^{j \mu}(1-\varepsilon)^{-1 / 2} \geqq \lambda^{-\mu}\left(\frac{t}{r_{m}}\right)^{\mu}(1-\varepsilon)^{-1 / 2} \geqq(1-\varepsilon)^{-1}\left(\frac{t}{r_{m}}\right)^{\mu},
$$

as well as

$$
\frac{\phi(t)}{\phi\left(r_{m}\right)} \leqq \frac{\phi\left(\lambda^{j+1} r_{m}\right)}{\phi\left(r_{m}\right)} \leqq \lambda^{(j+1) \mu}(1+\varepsilon)^{1 / 2} \leqq \lambda^{\mu}\left(\frac{t}{r_{m}}\right)^{\mu}(1+\varepsilon)^{1 / 2} \leqq(1+\varepsilon)\left(\frac{t}{r_{m}}\right)^{\mu} .
$$

We have thus proved that (7.2) implies (7.3); these relations may be used with

$$
B=l, \quad \varepsilon=1 / l \quad(l=2,3,4, \ldots) .
$$

As $l \rightarrow+\infty$ by integral values we are led to a sequence $m_{l}$ (which may be assumed to be strictly increasing and unbounded) such that the inequalities

$$
r_{m} / l \leqq t \leqq l r_{m}, \quad m \geqq m_{l},
$$

imply

$$
\left(\frac{l}{l+1}\right)\left(\frac{t}{r_{m}}\right)^{\mu} \leqq \frac{\phi(t)}{\phi\left(r_{m}\right)} \leqq\left(1+\frac{1}{l}\right)\left(\frac{t}{r_{m}}\right)^{\mu} .
$$

Consider successively the intervals

and set

$$
m_{l} \leqq m<m_{l+1} \quad(l=2,3,4, \ldots)
$$

$$
R_{m}^{\prime}=\max \left(l^{-1} r_{m},\left(r_{m}\right)^{1 / 2}\right), \quad R_{m}^{\prime \prime}=l r_{m}, \tilde{\varepsilon}_{m}=1 / l .
$$

The sequences $\left\{R_{m}^{\prime}\right\},\left\{R_{m}^{n}\right\},\left\{\tilde{\varepsilon}_{m}\right\}$ thus defined satisfy the relations (7.4) and, by using (7.13) in (7.11) and (7.12), we see that (7.5) implies (7.6).

The particular choice $\phi=T$ and (6.7) show that (2.8) implies (2.9).

8. Proof of (2.10) and (2.11). In view of the symmetrical roles played by $u$ and $v$, it is convenient to write $w$ instead of $u$ or $v$ and an $N(t)$ instead of $N(t, 1 / f)$ or $N(t, f)$. It is clear that the interpretation $u=w$ is to be associated with $N(t)=N(t, 1 / f)$ and $v=w$ with $N(t)=N(t, f)$.

We prove first

$$
\limsup _{m \rightarrow \infty} \frac{N\left(r_{m}\right)}{T\left(r_{m}\right)} \leqq w ;
$$

this is not obvious because of the presence of the exceptional set $\mathscr{E}$ in the definitions (1.9).

If (8.1) were wrong, we would have for a suitable $\kappa>0$,

$$
N\left(r_{m}\right) / T\left(r_{m}\right)>w+\kappa,
$$


as $m$ runs through values of some unbounded sequence $\mathscr{M}$. Consider now the intervals

$$
r_{m} \leqq t \leqq\left(\frac{w+\kappa}{w+\frac{1}{2} \kappa}\right)^{1 / \mu} r_{m} \quad(m \in \mathscr{M})
$$

let $\mathscr{U}$ be their union and notice that $\mathscr{U}$ is certainly not a set of density zero.

If $t \in \mathscr{U}$, we deduce from (8.2), (8.3), (2.9) and the fact that $N(t)$ is nondecreasing:

$$
\begin{gathered}
N(t)>(w+\kappa) \frac{T\left(r_{m}\right)}{T(t)} T(t) \geqq\left(w+\frac{\kappa}{2}\right) \frac{T(t)}{1+\tilde{\varepsilon}_{m}} \quad(m \in \mathscr{M}), \\
\liminf _{t \rightarrow \infty: t \in \mathscr{Q}} \frac{N(t)}{T(t)} \geqq w+\frac{\kappa}{2} .
\end{gathered}
$$

As this contradicts (1.9) we see that (8.1) must hold.

We now turn to the proof of

$$
\lim _{m \rightarrow \infty} \frac{N\left(r_{m}\right)}{T\left(r_{m}\right)}=w .
$$

In view of (8.1), if (8.4) does not hold, we have $w>0$ and, for a suitable $\kappa(0<\kappa<w)$ and all $m$ belonging to some unbounded sequence $\mathscr{M}$,

$$
N\left(r_{m}\right) / T\left(r_{m}\right)<w-\kappa .
$$

Put

$$
\xi=\left\{\frac{w-\kappa}{w-\frac{1}{2} \kappa}\right\}^{1 / \mu},
$$

and notice that, if $t$ lies in the interval $\left[\xi r_{m}, r_{m}\right],(8.5)$ and (2.9) yield

$$
\begin{aligned}
N(t) & \leqq N\left(r_{m}\right)<(w-\kappa) \frac{T\left(r_{m}\right)}{T(t)} T(t) \leqq\left(w-\frac{1}{2} \kappa\right) T(t)\left(1+\tilde{\varepsilon}_{m}\right) \\
& \leqq\left(w-\frac{1}{3} \kappa\right) T(t) \quad\left(m \in \mathscr{M}, m>m_{0}\right) .
\end{aligned}
$$

Consider (8.6) in the case $w=u$ and use it to estimate the first integral in the right-hand side of (4.5). We see at once that, given $\varepsilon>0$, this integral cannot exceed

$$
\begin{aligned}
(w+\varepsilon)\left\{\int_{\rho_{m}^{\prime}}^{\xi r_{m}}+\right. & \left.\int_{r_{m}}^{\rho_{m}^{*}}\right\} T(t) P\left(t, r_{m}, \gamma_{m}\right) d t \\
& +\left(w-\frac{1}{3} \kappa\right) \int_{\xi r_{m}}^{r_{m}} T(t) P\left(t, r_{m}, \gamma_{m}\right) d t \quad\left(m \in \mathscr{M}, m>m_{0}(\varepsilon)\right) .
\end{aligned}
$$

Hence we deduce from (4.5)

$$
\begin{aligned}
(1-\varepsilon) T\left(r_{m}\right) \leqq & (u+\varepsilon) \int_{\rho_{m}^{\prime}}^{\rho_{m}^{*}} T(t) P\left(t, r_{m}, \gamma_{m}\right) d t \\
& +(v+\varepsilon) \int_{\rho_{m}^{\prime}}^{\rho_{m}^{\prime \prime}} T(t) P\left(t, r_{m}, \pi-\gamma_{m}\right) d t \\
& -\frac{\kappa}{3} T\left(\xi r_{m}\right) \int_{\xi}^{1} P\left(x, 1, \gamma_{m}\right) d x \quad\left(m \in \mathscr{M}, m>m_{0}\right) .
\end{aligned}
$$


Since

$$
\lim _{m \rightarrow \infty} \frac{T\left(\xi r_{m}\right)}{T\left(r_{m}\right)}=\xi^{\mu}
$$

it is clear that if we let $m \rightarrow+\infty$ by values of $\mathscr{M}$, the method used in $\S 6$ leads to

$$
(1-\varepsilon) \leqq(u+\varepsilon) \frac{\sin \beta \mu}{\sin \pi \mu}+(v+\varepsilon) \frac{\sin (\pi-\beta) \mu}{\sin \pi \mu}-\frac{\kappa}{3} \xi^{\mu} \int_{\xi}^{1} P(x, 1, \beta) d x .
$$

Letting $\varepsilon \rightarrow 0$, we obtain again (6.18) which is incompatible with (6.6). Hence (8.5) cannot hold and we thus obtain (8.4) in the case $w=u$. The case $w=v$ is covered by the same arguments and requires no special study.

Before proving the relations (2.10) we notice that for $\lambda$ fixed $(0<\lambda<+\infty)$, two applications of (2.9) show that, if $t$ satisfies (2.8), then

$$
\left(t / \lambda r_{m}\right)^{\mu}\left(1+\tilde{\varepsilon}_{m}\right)^{-2} \leqq T(t) / T\left(\lambda r_{m}\right) \leqq\left(t / \lambda r_{m}\right)^{\mu}\left(1+\tilde{\varepsilon}_{m}\right)^{2}
$$

From (8.8) we deduce that, for every fixed $\lambda>0$, the sequence $\left\{\lambda r_{m}\right\}$ is a sequence of Pólya peaks of $T(r)$. Hence (8.4) also implies

$$
\lim _{m \rightarrow+\infty} \frac{N\left(\lambda r_{m}\right)}{T\left(\lambda r_{m}\right)}=w
$$

for every fixed $\lambda>0$.

Assume first that $w \neq 0$. Then (8.9) yields

$$
\lim _{m \rightarrow \infty} \frac{N\left(\lambda r_{m}\right) T\left(r_{m}\right)}{T\left(\lambda r_{m}\right) N\left(r_{m}\right)}=1
$$

and since $\left\{T\left(r_{m}\right) / T\left(\lambda r_{m}\right)\right\} \rightarrow \lambda^{-\mu}$, we deduce that

$$
\lim _{m \rightarrow \infty} \frac{N\left(\lambda r_{m}\right)}{N\left(r_{m}\right)}=\lambda^{\mu}
$$

for every fixed $\lambda>0$.

Since $N(t)$ is nondecreasing, (8.10) enables us to apply Lemma 4 with $\phi(t)=N(t)$. We thus obtain

If $w \neq 0$ and if

$$
R_{m}^{\prime} \leqq t \leqq R_{m}^{\prime \prime} \quad\left(m \geqq m_{0}\right)
$$

then

$$
\left(t / r_{m}\right)^{\mu}\left(1+\tilde{\varepsilon}_{m}\right)^{-1} \leqq N(t) / N\left(r_{m}\right) \leqq\left(t / r_{m}\right)^{\mu}\left(1+\tilde{\varepsilon}_{m}\right) .
$$

The arguments do not prove that the quantities $R_{m}^{\prime}, R_{m}^{\prime \prime}, \tilde{\varepsilon}_{m}$ in (8.11) and (8.12) coincide with those in (2.8) and (2.9). This is unimportant because an inspection of (8.11) and (8.12) (and of their analogues (2.8) and (2.9)) show that these relations remain true if $\tilde{\varepsilon}_{m}$ and $R_{m}^{\prime}$ are replaced by larger values and $R_{m}^{\prime \prime}$ by a smaller value. Hence any conflict can always be resolved by a change of notation. 
From (2.9) and (8.12), we deduce

$$
\frac{N\left(r_{m}\right)}{T\left(r_{m}\right)}\left(1+\tilde{\varepsilon}_{m}\right)^{-2} \leqq \frac{N(t)}{T(t)} \leqq \frac{N\left(r_{m}\right)}{T\left(r_{m}\right)}\left(1+\tilde{\varepsilon}_{m}\right)^{2},
$$

provided $t$ lies in the interval (8.11) and $w \neq 0$.

Hence (8.4) and, if necessary, a change of notation lead to the relations (2.10).

The case $w=0$ requires a slightly different treatment since it is no longer certain that (8.12) is true.

Let $l>1$ be an integer and let

$$
l^{-1} r_{m} \leqq t \leqq l r_{m}
$$

Since $N(t)$ and $T(t)$ are nondecreasing, we obtain, in view of (2.9) and (8.9)

$$
0 \leqq \frac{N(t)}{T(t)} \leqq \frac{N\left(l r_{m}\right)}{T\left(l^{-1} r_{m}\right)} \leqq \frac{N\left(l r_{m}\right)}{T\left(l r_{m}\right)} l^{2 \mu}\left(1+\tilde{\varepsilon}_{m}\right)^{2}<\frac{1}{l}
$$

provided $m$ exceeds a suitable bound $m_{l}$.

The arguments following formula (7.12) show that (8.14) yields (2.10) in the special case $w=0$. Hence (2.10) is proved in all cases.

In order to pass from (2.10) to (2.11), we use a straightforward tauberian argument.

Let $1<\lambda<2$; start from the obvious relations

$$
n(t) \frac{\lambda-1}{\lambda} \leqq \int_{t}^{\lambda t} \frac{n(x)}{x} d x=N(\lambda t)-N(t)
$$

and confine $t$ to the intervals $\left[2 R_{m}^{\prime}, R_{m}^{n} / 2\right]$, so that $\lambda t$ lies in $\left[2 R_{m}^{\prime}, R_{m}^{n}\right]$.

From (8.15) and (2.10) we deduce

$$
n(t) \frac{\lambda-1}{\lambda} \leqq\left(w+\tilde{\varepsilon}_{m}\right) T(\lambda t)-\left(w-\tilde{\varepsilon}_{m}\right) T(t) \leqq w[T(\lambda t)-T(t)]+2 \tilde{\varepsilon}_{m} T(\lambda t),
$$

and by (2.9)

$$
\begin{aligned}
\frac{n(t)}{T(t)} \frac{\lambda-1}{\lambda} & \leqq w\left\{\lambda^{\mu}\left(1+\tilde{\varepsilon}_{m}\right)^{2}-1\right\}+2 \tilde{\varepsilon}_{m} \lambda^{\mu}\left(1+\tilde{\varepsilon}_{m}\right)^{2}, \\
\frac{n(t)}{T(t)} & \leqq \lambda w \frac{\lambda^{\mu}-1}{\lambda-1}+\frac{11 \tilde{\varepsilon}_{m} \lambda^{\mu+1}}{\lambda-1} \quad\left(m>m_{0}, \tilde{\varepsilon}_{m}<1\right) .
\end{aligned}
$$

If we set $\lambda=1+\left(\tilde{\varepsilon}_{m}\right)^{1 / 2}$, let $m \rightarrow+\infty$ and notice that $\left(\lambda^{\mu}-1\right) /(\lambda-1) \rightarrow \mu$, we deduce from (8.16)

$$
n(t) / T(t) \leqq \mu w+\zeta_{m} \quad\left(2 R_{m}^{\prime} \leqq t \leqq \frac{1}{2} R_{m}^{\prime \prime}\right),
$$

where $\lim _{m \rightarrow \infty} \zeta_{m}=0$.

A lower bound for $n(t) / T(t)$ may be obtained by the same method: start from

$$
N(t)-N(\lambda t) \leqq n(t)(1-\lambda) / \lambda \quad\left(\frac{1}{2}<\lambda<1\right),
$$


set $\lambda=1-\left(\tilde{\varepsilon}_{m}\right)^{1 / 2}$ and let $m \rightarrow+\infty$. The resulting inequality and (8.17) lead to (2.11) after an obvious modification of the definitions of $R_{m}^{\prime}, R_{m}^{\prime \prime}$ and $\tilde{\varepsilon}_{m}$.

\section{Proof of assertion I of Theorem 2. Let}

$$
\tau_{1}, \tau_{2}, \tau_{3}, \ldots
$$

be an infinite sequence containing all the finite deficient values of $g(z)$. We assume that some member of $(9.1)$, say $\tau_{1}$, has a positive deficiency:

$$
\delta_{1}=\delta\left(\tau_{1}, g\right)>0
$$

this is no restriction because, if $g(z)$ has no finite deficient values, $(2.17)$ is trivial.

Let $q>0$ be an integer and let

$$
H_{q}(z)=\prod_{j=1}^{q}\left(g(z)-\tau_{j}\right) .
$$

It is well known that, for all $r \geqq 0$,

$$
\sum_{j=1}^{q} m\left(r, \frac{1}{g(z)-\tau_{j}}\right) \leqq m\left(r, \frac{1}{g^{\prime}}\right)+m\left(r, \frac{H_{q}^{\prime}}{H_{q}}\right)+K(q),
$$

where the constant $K(q)$ depends only on the first $q$ members of (9.1). [The inequality (9.3) is implicit in the proof of Nevanlinna's second fundamental theorem. An explicit formulation and proof will be found in [5, pp. 284-285].]

The classical estimates of the logarithmic derivative $[10$, p. 40] show that

$$
\begin{aligned}
m\left(r, H_{q}^{\prime} / H_{q}\right) \leqq 10\{\log T(r, g)+\log r\} & \left(r \notin \mathscr{E}, r>r_{0}\right), \\
m\left(r, g^{\prime} / g\right) \leqq 10\{\log T(r, g)+\log r\} & \left(r \notin \mathscr{E}, r>r_{0}\right),
\end{aligned}
$$

where $\mathscr{E}$ is a set of finite measure. In (9.4) the finite quantity $r_{0}$ depends on $g, q$ and the sequence (9.1). On the other hand, it is important for our purposes to observe with Hayman [10, p. 41] that the set $\mathscr{E}$ depends only on $T(r, g)$ and not on $q$ or (9.1).

By assumption $\mu>0$ so that (9.3), (9.4) and (9.5) yield

$$
\begin{gathered}
\sum_{j=1}^{q} m\left(r, \frac{1}{g-\tau_{j}}\right) \leqq m\left(r, \frac{1}{g^{\prime}}\right)+10\left(1+\frac{2}{\mu}\right) \log T(r) \\
\left(T(r)=T(r, g), r \notin \mathscr{E}, r>r_{0}(q)\right), \\
m\left(r, g^{\prime} / g\right) \leqq 10(1+2 / \mu) \log T(r) \quad\left(r \notin \mathscr{E}, r>r_{0}\right)
\end{gathered}
$$

The latter inequality and the familiar relations

$$
m\left(r, g^{\prime}\right) \leqq m\left(r, g^{\prime} / g\right)+m(r, g), \quad N\left(r, g^{\prime}\right) \leqq 2 N(r, g)
$$

imply

$$
T\left(r, g^{\prime}\right)=T_{1}(r) \leqq T(r)+N(r, g)+10(1+2 / \mu) \log T(r) \quad\left(r \notin \mathscr{E}, r>r_{0}\right),
$$


and in view of $\delta(\infty, g)=1$

$$
T_{1}(r) \leqq T(r)(1+o(1)) \quad(r \rightarrow+\infty, r \notin \mathscr{E}) .
$$

By (9.6) and the first fundamental theorem we obtain

$$
N\left(r, \frac{1}{g^{\prime}}\right)+\sum_{j=1}^{q} m\left(r, \frac{1}{g-\tau_{j}}\right) \leqq T_{1}(r)+10\left(1+\frac{2}{\mu}\right) \log T(r)+K
$$

$$
\left(r \notin \mathscr{E}, r>r_{0}(q)\right),
$$

and hence, in view of (9.2),

$$
\frac{1}{2} \delta_{1} T(r) \leqq T_{1}(r) \quad\left(r \notin \mathscr{E}, r>r_{0}\right) .
$$

Using (9.11) in (9.10), we find

$$
\frac{N\left(r, \frac{1}{g^{\prime}}\right)}{T_{1}(r)}+\frac{T(r)}{T_{1}(r)} \sum_{j=1}^{q} \frac{m\left(r, \frac{1}{g-\tau_{j}}\right)}{T(r)} \leqq 1+o(1) \quad(r \rightarrow+\infty, r \notin \mathscr{E}) .
$$

Consider (2.16) and let $r \rightarrow+\infty$ by a sequence of values $\left\{\tilde{r}_{m}\right\}$ such that $\tilde{r}_{m} \notin \mathscr{E}$ and

$$
\lim _{m \rightarrow \infty} \frac{N\left(\tilde{r}_{m}, 1 / g^{\prime}\right)}{T_{1}\left(\tilde{r}_{m}\right)}=u\left(g^{\prime}\right) .
$$

Hence (9.12), with $r=\tilde{r}_{m}$, and (9.9) yield

$$
u\left(g^{\prime}\right)+\sum_{j=1}^{q} \delta\left(\tau_{j}, g\right) \leqq 1,
$$

and therefore

$$
u\left(g^{\prime}\right)+\sum_{j=1}^{\infty} \delta\left(\tau_{j}, g\right) \leqq 1
$$

The second inequality in (9.8) and (9.11) imply

$$
\begin{aligned}
\frac{N\left(r, g^{\prime}\right)}{T_{1}(r)} & \leqq \frac{4}{\delta_{1}} \frac{N(r, g)}{T(r, g)} \quad\left(r \notin \mathscr{E}, r>r_{0}\right), \\
v\left(g^{\prime}\right) & \leqq \frac{4}{\delta_{1}}(1-\delta(\infty, g))=0 .
\end{aligned}
$$

From (9.9) and (9.11), we deduce that the lower order of $g^{\prime}(z)$ coincides with the lower order of $g(z)$. Hence, applying assertion I of Theorem 1 to the function $g^{\prime}$, and using (9.14), we find

$$
\sin \pi \mu \leqq u\left(g^{\prime}\right) .
$$

In view of the assumption $\delta(\infty, g)=1,(9.13)$ and $(9.15)$ yield

$$
\Delta(g)=\delta(\infty, g)+\sum_{j=1}^{\infty} \delta\left(\tau_{j}, g\right) \leqq 1+(1-\sin \pi \mu)
$$

and (2.17) follows. 
10. Proof of assertion II of Theorem 2. By assumption, equality holds in (9.16) so that

$$
\sum_{j=1}^{\infty} \delta\left(\tau_{j}, g\right)=1-\sin \pi \mu
$$

which used in (9.13) yields $u\left(g^{\prime}\right) \leqq \sin \pi \mu$ and, in view of (9.15), $u\left(g^{\prime}\right)=\sin \pi \mu$. This equality and (9.14) prove (2.18). Hence

$$
\sin ^{2} \pi \mu=u^{2}\left(g^{\prime}\right)+v^{2}\left(g^{\prime}\right)-2 u\left(g^{\prime}\right) v\left(g^{\prime}\right) \cos \pi \mu \quad\left(v\left(g^{\prime}\right)=0\right) .
$$

Since $\frac{1}{2}<\mu<1$ we also have $u\left(g^{\prime}\right)<1$ and part II of Theorem 1 may be applied to the function $g^{\prime}$; this yields (2.20), (2.21) and (2.22).

In order to complete the proof of Theorem 2, we now study the ratio $T(t) / T_{1}(t)$ on the set $V_{\sigma}\left(\left\{r_{m}\right\}\right)$ introduced in (2.12).

Given $\varepsilon(0<\varepsilon<1-\sin \pi \mu)$, choose $q$ so large that

$$
\sum_{j=1}^{q} m\left(t, \frac{1}{g-\tau_{j}}\right)>(1-\sin \pi \mu-\varepsilon) T(t) \quad\left(t>t_{0}(\varepsilon)\right) ;
$$

this is possible by the definition of deficiency and (10.1).

Given $t$, denote by $t^{\prime}=t^{\prime}(t), t^{\prime \prime}=t^{\prime \prime}(t)$, points such that

$$
t-1 \leqq t^{\prime} \leqq t, \quad t \leqq t^{\prime \prime} \leqq t+1, \quad t^{\prime} \notin \mathscr{E}, \quad t^{\prime \prime} \notin \mathscr{E} .
$$

If $t \geqq t_{0}$, such points $t^{\prime}, t^{\prime \prime}$ always exist because $\mathscr{E}$ is of finite measure. Notice also that $t \in V_{\sigma}\left(\left\{r_{m}\right\}\right)\left(t \geqq t_{0}\right)$ implies

$$
t^{\prime} \in V_{\sigma+1}\left(\left\{r_{m}\right\}\right), \quad t^{\prime \prime} \in V_{\sigma+1}\left(\left\{r_{m}\right\}\right)
$$

Hence, given $\varepsilon>0$, we deduce from (2.21)

$$
(-\varepsilon+\sin \pi \mu) T_{1}\left(t^{\prime \prime}\right) \leqq N\left(t^{\prime \prime}, 1 / g^{\prime}\right) \quad\left(t^{\prime \prime}=t^{\prime \prime}(t), t \in V_{\sigma}\left(\left\{r_{m}\right\}\right), t>t_{0}(\varepsilon)\right),
$$

and from (10.2)

$$
\sum_{j=1}^{q} m\left(t^{\prime \prime}, \frac{1}{g-\tau_{j}}\right)>(1-\sin \pi \mu-\varepsilon) T\left(t^{\prime \prime}\right) .
$$

Similarly (9.12) holds with $r=t^{\prime \prime}$ and, in view of (10.4) and (10.5), leads to

$$
\begin{gathered}
(-\varepsilon+\sin \pi \mu) T_{1}\left(t^{\prime \prime}\right)+(1-\sin \pi \mu-\varepsilon) T\left(t^{\prime \prime}\right) \leqq(1+\varepsilon) T_{1}\left(t^{\prime \prime}\right), \\
\frac{T(t)}{T_{1}\left(t^{\prime \prime}\right)} \leqq \frac{1-\sin \pi \mu+2 \varepsilon}{1-\sin \pi \mu-\varepsilon} \quad\left(t \in V_{\sigma}\left(\left\{r_{m}\right\}\right), t>t_{0}(\varepsilon)\right) .
\end{gathered}
$$

Using (2.20), we eliminate $t^{\prime \prime}$ from (10.6):

$$
\begin{aligned}
\frac{T(t)}{T_{1}(t)} & \leqq\left(1+\tilde{\varepsilon}_{m}\right)^{2}\left(\frac{t^{\prime \prime}}{t}\right)^{\mu}\left(1+3 \varepsilon[1-\sin \pi \mu-\varepsilon]^{-1}\right) \\
& \leqq(1+\varepsilon)\left(1+3 \varepsilon[1-\sin \pi \mu-\varepsilon]^{-1}\right),
\end{aligned}
$$

provided $t$ is large enough (and $t \in V_{\sigma}\left(\left\{r_{m}\right\}\right)$ ). 
A lower bound for $T(t) / T_{1}(t)$ follows readily from (9.9), which holds for $r=t^{\prime}(t)$ :

$$
T_{1}\left(t^{\prime}\right) \leqq T\left(t^{\prime}\right)(1+\varepsilon) \leqq T(t)(1+\varepsilon) \quad\left(t \geqq t_{0}\right) .
$$

As in (10.6), we eliminate $t^{\prime}$ by using $(2.20)$ and find

$$
(1+\varepsilon)^{-2} \leqq\left(1+\tilde{\varepsilon}_{m}\right)^{-2}\left(\frac{t^{\prime}}{t}\right)^{\mu}(1+\varepsilon)^{-1} \leqq \frac{T(t)}{T_{1}(t)} \quad\left(t \in V_{\sigma}\left(\left\{r_{m}\right\}\right), t>t_{0}(\varepsilon)\right)
$$

From (10.7) and (10.8), we deduce that for $\sigma(>1)$ fixed,

$$
T(t) / T_{1}(t) \rightarrow 1
$$

as $t \rightarrow+\infty, t \in V_{\sigma}\left(\left\{r_{m}\right\}\right)$. With minor modifications, the arguments of $\S 7$ (following formula (7.12)) show that (10.9) is equivalent to (2.23). A change of notation (such as the one described after formula (8.12)) enables us to ensure that $R_{m}^{\prime}, R_{m}^{\prime \prime}$ and $\tilde{\varepsilon}_{m}$ have the same meaning throughout the statement of Theorem 2 .

11. Proof of Corollary 1.1. From part I of Theorem 1 we deduce

$$
u^{2}+v^{2}-2 u v \cos \pi \mu \geqq \sin ^{2} \pi \mu,
$$

and

$$
\cos \pi \mu<u<1, \quad \cos \pi \mu<v<1
$$

The latter inequalities imply

$$
1-\cos \pi \mu>|u-v| .
$$

Returning to (11.1) and using (11.2), we find

$$
\begin{aligned}
2 u v(1-\cos \pi \mu) & \geqq \sin ^{2} \pi \mu-(u-v)^{2}>\sin ^{2} \pi \mu-(1-\cos \pi \mu)^{2} \\
& =2 \cos \pi \mu(1-\cos \pi \mu), \\
2 u v & >2 \cos \pi \mu \geqq \cos \pi \mu+\cos \pi \Lambda .
\end{aligned}
$$

The inequality (11.1) may be rewritten as

$$
u^{2}+v^{2}-2 u v \cos \pi \Lambda \geqq \sin ^{2} \pi \mu+2 u v(\cos \pi \mu-\cos \pi \Lambda) .
$$

Assume Corollary 1.1 to be wrong. Then $\cos \pi \mu-\cos \pi \Lambda>0$, and (11.3) yields

$$
2 u v(\cos \pi \mu-\cos \pi \Lambda)>\cos ^{2} \pi \mu-\cos ^{2} \pi \Lambda,
$$

which, used in (11.4), leads to

$$
u^{2}+v^{2}-2 u v \cos \pi \Lambda>\sin ^{2} \pi \Lambda .
$$

This contradicts (2.25) and hence proves Corollary 1.1. 


\section{REFERENCES}

1. A. Edrei, The deficiencies of meromorphic functions of finite lower order, Duke Math. J. 31 (1964), 1-22.

2. - Sums of deficiencies of meromorphic functions, J. Analyse Math. 14 (1965), 79-107.

3. - Sums of deficiencies of meromorphic functions. II, J. Analyse Math. 19 (1967), 53-74.

4. A. Edrei and W. H. J. Fuchs, On the growth of meromorphic functions with several deficient values, Trans. Amer. Math. Soc. 93 (1959), 292-328.

5. - Valeurs déficientes et valeurs asymptotiques des fonctions méromorphes, Comment. Math. Helv. 33 (1959), 258-295.

6. - The deficiencies of meromorphic functions of order less than one, Duke Math. J. 27 (1960), 233-250.

7. - Bounds for the number of deficient values of certain classes of functions, Proc. London Math. Soc. 12 (1962), 315-344.

8. - Tauberian theorems for a class of meromorphic functions with negative zeros and positive poles, Proc. Internat. Conf. Function Theory, Erevan, 1965, "Nauka”, Moscow, 1966, pp. 339-358.

9. G. H. Hardy and J. E. Littlewood, Notes on the theory of series. XI. On Tauberian Theorems, Proc. London Math. Soc. 30 (1930), 23-37.

10. W. K. Hayman, Meromorphic functions, Clarendon Press, Oxford, 1964.

11. A. Pfluger, Zur Defektrelation ganzer Funktionen endlicher Ordnung, Comment. Math. Helv. 19 (1946-1947), 91-104.

12. G. Valiron, Sur les fonctions entières d'ordre fini et d'ordre nul, et en particulier les fonctions à correspondance régulière, Ann. Fac. Sci. Univ. Toulouse 5 (1913), 117-257.

13. H. Wittich, Neuere Untersuchungen über eindeutige analytische Funktionen, SpringerVerlag, Berlin, 1955.

SYRACUSE UNIVERSITY, SYRACUSE, NeW YoRK 